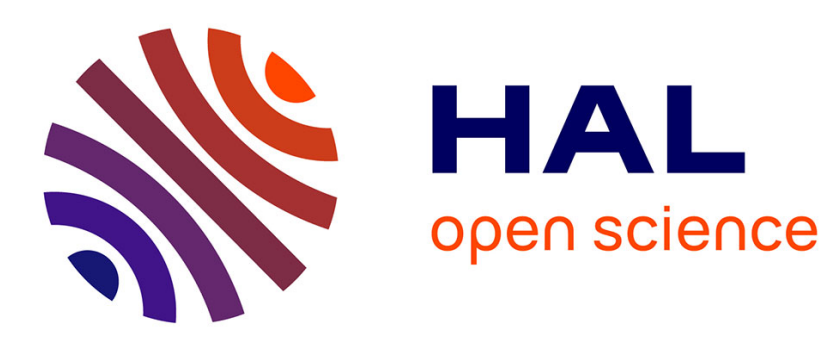

\title{
A water hammer analysis of pressure and flow in the voice production system \\ D. Sciamarella, G. Artana
}

\section{To cite this version:}

D. Sciamarella, G. Artana. A water hammer analysis of pressure and flow in the voice production system. Speech Communication, 2009, 51 (4), pp.344. 10.1016/j.specom.2008.10.004 . hal-00509239

\section{HAL Id: hal-00509239 \\ https://hal.science/hal-00509239}

Submitted on 11 Aug 2010

HAL is a multi-disciplinary open access archive for the deposit and dissemination of scientific research documents, whether they are published or not. The documents may come from teaching and research institutions in France or abroad, or from public or private research centers.
L'archive ouverte pluridisciplinaire HAL, est destinée au dépôt et à la diffusion de documents scientifiques de niveau recherche, publiés ou non, émanant des établissements d'enseignement et de recherche français ou étrangers, des laboratoires publics ou privés. 


\section{Accepted Manuscript}

A water hammer analysis of pressure and flow in the voice production system

D. Sciamarella, G. Artana

PII:

S0167-6393(08)00162-3

DOI:

10.1016/j.specom.2008.10.004

Reference:

SPECOM 1759

To appear in:

Speech Communication

Received Date:

6 June 2008

Revised Date:

17 October 2008

Accepted Date:

17 October 2008

Please cite this article as: Sciamarella, D., Artana, G., A water hammer analysis of pressure and flow in the voice production system, Speech Communication (2008), doi: 10.1016/j.specom.2008.10.004

This is a PDF file of an unedited manuscript that has been accepted for publication. As a service to our customers we are providing this early version of the manuscript. The manuscript will undergo copyediting, typesetting, and review of the resulting proof before it is published in its final form. Please note that during the production process errors may be discovered which could affect the content, and all legal disclaimers that apply to the journal pertain. 


\title{
A water hammer analysis of pressure and flow in the voice production system
}

\author{
D. Sciamarella ${ }^{a}$ G. Artana ${ }^{b}$ \\ ${ }^{\mathrm{a}} L I M S I-C N R S$ \\ BP 133 - F 91403 ORSAY Cedex, FRANCE \\ ${ }^{\mathrm{b}}$ LFD, Facultad de Ingeniería, Universidad de Buenos Aires \\ CONICET
}

\begin{abstract}
The sudden pressure rise produced by glottal closure in the subglottal tract during vocal fold oscillation causes a flow transient which can be computed as a water hammer effect in engineering. In this article, we present a basic water hammer analysis for the trachea and the supralaryngeal tract under conditions which are analogue to those operating during voice production. This approach allows predicting both, the intra-oral and intra-tracheal pressure fluctuations induced by vocal fold motion, as well as the airflow evolution throughout the phonatory system.
\end{abstract}

Key words: voice production, water hammer, speech synthesis PACS: 43.70-h, 43.70.Bk, 43.28.Ra

\section{Introduction}

Water hammer, also referred to as pressure surges or fluid transients in pipelines, is an oscillatory form of unsteady flow generated by sudden changes due to the rapid closing or opening of valves, or due to a pumping action. The term 'water hammer' was first coined to refer to the large transient pressure variations causing damage to liquid-filled pipe systems, but it later acquired a generality which nowadays makes it applicable to all types of transient flow in pipelines. Conventional water hammer analyses provide information under operational conditions on two unknowns: pressure and velocity within a pipe system. The pipe system is ordinarily supposed to be rigidly anchored and the source of fluid transients is usually handled as a kinematic condition for the flow, as is common in aero-elasticity problems. Effects such as unsteady friction, acoustic radiation to the surroundings or fluid structure interaction are not taken into account in the standard theory of water hammer, but have 
been considered in the context of a more general approach $[1,2]$. In particular, fluid structure interaction (FSI) can be presented as an extension of conventional water hammer theory that includes mechanisms acting both all along the entire pipe (such as axial stresses in the pipe) and at specific points in the pipe system (such as unrestrained valves, bends or tees). The water hammer theory has been proposed to account for a number of effects in biofluids under mechanical stress, as in the case of the origin of Korotkoff sounds during blood pressure measurement [3,4], or the development of a fluid-filled cavity or syrinx within the spinal cord [5].

In the voice production system, the human vocal folds act as a valve [6] which induces pressure waves at a specific 'point' in the airways (the glottis), through successive compressing and decompressing actions (the glottis opens and closes repeatedly). Ishizaka was probably the first to advocate in 1976 the application of the water hammer theory to the phonatory system, when discussing the input acoustic impedance looking into the trachea [7]. More recently, the water hammer theory was invoked in the context of tracheal wall motion detection [8]. The analogy between the system trachea-glottis and the system pipe-valve is natural and direct. Extending the analogy to the supraglottal airways is however possible, even if these have a complex shape and behaviour, in particular during speech production. In fact, the vocal tract is often modelled as a tube of variable cross sections. This allows integration of the whole phonatory system in a single pipeline intercepted by a valve, and renders the water hammer theory applicable from the trachea to the mouth. To our knowledge, the water hammer theory has not been previously implemented to predict simultaneoulsy intra-tracheal and intra-oral pressure and flow. The aim of this work is to remedy this omission and to highlight the interesting aspects that result from applying the water hammer theory to the complete phonatory system.

This task can be performed at different levels of complexity according to the version of the water hammer theory that is applied, and according to the settings used to define the pipe system. Here we will adopt a static pipeline and we will implement the two-equation version (according to the nomenclature used in [2]) sometimes referred to as basic water hammer, in order to reproduce the results obtained with the customary description of the vocal tract. For these purposes, the action of the glottis will be translated into a valve operation that generates a fluid transient in the pipe system, with a prescribed open-close manœuvre repeated at a constant frequency (the fundamental frequency of the voiced sound). Such a prescription is typical of forced oscillation glottal models [9]. This simple treatment naturally excludes the analysis of the conditions under which the self-sustained oscillations of the valve are initiated and maintained [10], since the structural dynamics is not computed but imposed. Indeed, in the extended water hammer theory, a self-oscillating glottis would rather be represented by a junction coupling with FSI, since the intravalvular 
pressure distribution causes the valve to move and the motion of the valve induces pressure waves in the fluid which in turn influence the motion of the valve. On the other hand, it might be particularly interesting to test the performance of a water hammer analysis on a dynamic (supralaryngeal) pipeline simulating speech production, i.e. the production of sequences of vowels and consonants.

In this work, we shall restrict our discussion to the production of a sustained vowel in the basic water hammer case. The analysis we present should therefore not be expected to improve the results obtained with other $1 \mathrm{D}$ voice production models, but to reproduce them by an alternate and well established procedure. This procedure has the advantage of providing a simplified but less indirect prediction of voice generation, in the sense that it does not require the introduction of a wave emitter model based on an acoustic analogy [11] as done in most vocal fold models.

The structure of the article is as follows. A background on voice production modelling is given in section 2 and a review of the basic concepts involved in water hammer theory is presented in section 3 . In section 4 , we present the application of the basic water hammer analysis to the production of a sustained vowel, under conditions and settings typically adopted in simplified vocal fold models. Section 5 shows the results of the analysis, with the prediction of intratracheal and intra-oral pressure and flow, compared to those obtained with a simple vocal fold model. Section 6 contains conclusions and perspectives.

\section{Background on voice production modelling}

In flow-induced sound production problems, the most common strategy is to consider sound generation and sound propagation separately, due to the large difference in time, length, and pressure scales involved in sound generation and transmission. Voice production is an example in which sound generation is more or less decoupled from the acoustic transport process, so that the flow equations can be conveniently tailored in each case. Fluid flow through the glottis (responsible for sound generation) behaves as a locally incompressible flow with thin but non negligible boundary layers, while fluid flow in the vocal tract (responsible for sound transmission) is compressible and reasonably described by the linearized Euler equation or simply by the plane wave equation. The standard procedure is therefore to solve for the flow and the acoustics separately, relying on some assumption that relates the flow through the source (the glottis) with the input sound pressure at the resonator (the supraglottal tract). The subglottal tract is seldom considered.

There have been many detailed studies of the flow and the acoustics involved in 
voice production. The flow picture through glottis and supraglottal tract used in the now classic two-mass model of Ishizaka and Flanagan [12] is the result of a considerable number of assumptions that, since then, have been thoroughly revisited and reconsidered. Much effort has been devoted in particular to correct the glottal flow model, which needs to capture flow separation and turbulent dissipation of the jet that is formed at the glottal exit $[13,14]$. On the other hand, the vocal tract is satisfactorily described in terms of linear acoustical properties. For the analysis of these acoustical properties, it is usually assumed that the wave motion in the tract is approximately planar, that the effects of viscosity are negligible, and that the vocal tract has rigid walls. Most 1D models represent the vocal tract as an acoustic tube of varying cross sectional area, approximated by a number of concatenated cylindrical sections. The properties of each section are included by using either a transmission line analogy (each section is represented by an equivalent $\mathrm{T}$ network) or a waveguide model (each section is a waveguide with boundary reflections observed at either end).

In this kind of voice production models, sound generation is predicted outside the flow, in the sense that the resonator subsystem accounts for the propagation of an already generated sound pressure, while the source subsystem accounts for non-acoustic flow motion. The stress is laid on vocal fold dynamics and sound propagation. An auxiliary model is therefore missing to predict sound generation at the connection between the glottis and the resonator. In other words, it becomes necessary to convert the volume flow at the glottal exit into an input sound pressure at the vocal tract entrance. This connecting model is necessarily a sound source model within the vocal fold model, introduced to express the generated pressure fluctuations in terms of the fluid dynamical variables at the glottal exit. The glottis is often approximated by a classic one dimensional monopole in a duct, an assumption that disregards the interaction between vortical structures and the velocity field; but further assumptions are still necessary to write the velocity fluctuations in terms of the glottal flow. Actually, there is some diversity in the final expression adopted for the input sound pressure in vocal fold models $[12,15,16]$. This expression involves a certain function of the glottal flow and its derivative (in general, a combination of linear, quadratic and piecewise linear functions). The reason for this diversity is that the sound field is a small perturbation of the flow and approximate solutions differ considerably depending on the assumptions that are involved.

If the fluid dynamical properties of the flow at the glottis are somehow experimentally assessed [17], and if they can be condensed in a law that contains the effects of the mechanical action of the vocal folds (or the glottal valve operation), the voice production problem bears more than a passing resemblance with basic water hammer problems. Notice that a water hammer analysis applied to voice production in this manner will depend, at the place of the sound 
source model mentioned above, on the characterization of the valve operation. However, we may say that in such an analysis the pressure fluctuations will be predicted inside the flow, i.e. without imposing a wave emitter model at the glottal exit. The water hammer theory allows to encompass sound generation and transmission along the phonatory system in a simple and straightforward calculation, provided that the action of the glottis as a flow-controlling valve can be prescribed.

\section{A review of basic water hammer}

Several authors have contributed to the present water hammer theory from the 19th century onwards [2]. Among them, Joukowsky [18] conducted a systematic study of the water distribution system in Moscow and derived a formula that bears his name:

$$
\Delta P=\rho c \Delta U
$$

This formula relates pressure fluctuations $(\Delta P)$ to velocity changes $(\Delta U)$ by a constant factor $\rho c$, where $\rho$ is the mass density of the fluid and $c$ the velocity of sound in the fluid. The expression for $c$ in the case of confined fluids was first derived to predict standing waves in musical instruments and pulsatile flows in blood vessels. For a compressible fluid in an elastic tube, $c$ depends on the bulk elastic modulus of the fluid $K$, on the elastic modulus of the pipe $E$, on the inner radius of the pipe $D_{0}$, and on its wall thickness $e$ :

$$
c=\sqrt{\frac{c_{0}^{2}}{1+\frac{D_{0} K}{e E}}}
$$

The water hammer equations are some version of the compressible fluid flow equations. The choice of the version is problem-dependent: basic water hammer neglects friction and damping mechanisms, classic water hammer takes into account fluid wall friction, extended water hammer allows for pipe motion and dynamic FSI [19-21]. The method of characteristics (MOC) is the standard numerical method for solving the water hammer equations [22]. For basic water hammer, the 1D equations are:

$$
\begin{array}{r}
\partial_{t} U+\frac{1}{\rho} \partial_{z} P=0 \\
\partial_{z} U+\frac{1}{\rho c^{2}} \partial_{t} P=0
\end{array}
$$


where $U$ is the velocity, $t$ is the time and $z$ is the distance along the pipe. In basic and classic water hammer problems, the valve operation inducing the transients is neither modelled nor computed: it is injected as input data. To this end, an accurate assessment of the pressure-flow-position behaviour of the valve is required.

The energy losses introduced in the system by the valve, are normally prescribed by means of an empirical law in terms of a loss coefficient. This coefficient, ordinarily determined under steady flow conditions, is known as the valve discharge coefficient, especially when the pipeline is terminated by the valve. It enables to quantify the flow response in terms of the valve action through a relationship between the flow rate and transvalvular pressure for each opening position of the valve. The discharge coefficient provides the critical piece of missing information for the water hammer analysis. Because the existing relationship between transvalvular pressure and flow rate is often a quadratic law type, the empirical coefficient is defined in terms of the squared flow rate.

For further reading, a detailed review of water hammer theory and practice is given in [23]. Fundamentals can be found in the classical textbook of Streeter and Wylie [24], as well as in [25].

\section{Water hammer analysis of a sustained vowel-like sound}

Four steps are necessary to adapt the basic water hammer procedure to the application we are concerned with:

(4.1) characterizing the pipeline;

(4.2) obtaining an expression for the discharge coefficient of the valve;

(4.3) prescribing the dynamics of the valve operation; and if necessary,

(4.4) defining some form of feedback.

Let us recall that this approach differs with respect to vocal fold models in the non minor point that the intravalvular dynamics is reduced to a single point where a mechanical action is prescribed. As a result, there is neither the need to reconcile fluid flows of different types, nor the need to place an acoustic wave emitter at the valve to account for sound generation. The scenario is a single fluid line responding to a valve operating at a specific point within the line. It is worth noting that water hammer flows are not restricted to the analysis of fluid lines $[26,27]$. It is our choice in this paper to present the simplest possible application of the water hammer theory to the voice production system. The 
only 'higher dimensional' ingredients that may be present in this scheme are those captured experimentally at the glottal level by the empirical coefficient that condenses the fluid dynamical effects of the valve operation.

\subsection{The pipeline}

The pipeline representing the airways involved in phonation can be quite complex if one includes a relatively realistic supraglottal configuration with, for instance, the cavity defined by the ventricular bands or the bifurcation leading to the nose. Here we will resume the settings and configurations used in simplified models to produce sustained vowels.

Let us consider a reservoir-pipe-valve-pipe system, corresponding respectively to the lungs, the trachea, the glottis and a non-bifurcated vocal tract. In water hammer practice, it is typical to characterize the geometry of the pipeline as a tube with piecewise constant cross sectional areas. This is how the pipeline was characterized by Korteweg to derive expression (2). These settings are also adopted in the usual acoustic-tube representation of the vocal tract. We will adopt the simple geometry sketched in figure 1, which roughly approximates with two sections the configuration of the vocal system necessary to produce a vowel with lip rounding (a very rough approximation of vowel $u$ ).

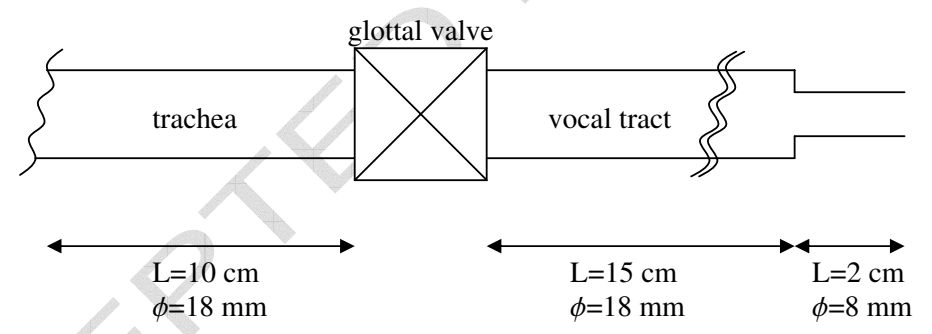

Fig. 1. Pipe-system geometry approximating the configuration of the vocal system used in the water hammer analysis.

The wall properties of the pipeline (elastic modulus, inner radius and thickness) are available in the literature, both for the trachea and the vocal tract. We calculate the wave speed using expression (2) with values for $E, D_{0}$ and $e$ following $[28,29]$ for the trachea and [30] for the vocal tract. The resulting wave speed in the vocal tract is almost equivalent to sound speed in air, whereas the wave speed in the trachea can be somewhat lower: $200 \mathrm{~m} / \mathrm{s}$ (in contrast to the $350 \mathrm{~m} / \mathrm{s}$ of the vocal tract). The (small) variations of the wave speed in the two vocal tract sections will be neglected, as in most simplified vocal fold models.

To set the boundary conditions at the pipeline inlet, we will consider a (lung) 
pressure of $800 \mathrm{~Pa}$. Downstream, at the pipeline outlet (lips) we will consider a discharge into an infinitely large space with an exit loss proportional to the square velocity of the flow at the outlet [31]. As mentioned in the introduction, the standard water hammer theory is not intended to predict pressure and flow outside the pipeline. This boundary condition is adopted to produce the correct level of loss within the pipe system. In fact, an estimate of the radiated speech sound would require some kind of wave emitter model at the tract exit. Inside the pipeline, the action of the valve and the cross sectional sudden variations of the pipe (in our example, the contraction at the second section of the tract near the exit) are set as additional internal boundary conditions.

The initial condition is an airflow starting from a quiescent state and a closed valve. The opening valve triggers the system response and maintains a forced periodic oscillation at a fixed frequency (in correspondence with a sustained voiced sound).

\subsection{The valve discharge coefficient}

The loss coefficient at the vocal folds may be obtained from in vitro experiments. Let us borrow the results obtained by Mongeau et al [17] in an experiment designed to verify the accuracy of a sound source model to predict the sound pressure generated by the glottis. In particular, let us retrieve the expression for the static orifice flow resistance as a function of the Reynolds number:

$$
C(R e)=c_{1} R e^{3}+c_{2} R e^{2}+c_{3} R e+c_{4}
$$

with $c_{1}=3.89 \times 10^{-11}, c_{2}=-2.18 \times 10^{-7}, c_{3}=4.19 \times 10^{-4}, c_{4}=5.75 \times 10^{-1}$. The Reynolds number is defined in terms of the volumetric flow rate $Q_{0}$, the tracheal dimensions and the kinematic viscosity.

Certainly, a dynamic (non static) loss coefficient would be more appropriate since the flow is not really quasi-steady at the vocal fold opening and closing instants. Such an unsteady loss coefficient could be gleaned, for instance, from an in silico experiment [14], but this would forbid a discretionary choice for the valve dynamics, which should strictly mimic the dynamics used in the numerical simulation. Again, this work retains the standard option in basic water hammer practice and assumes that the valve discharge coefficient measured under steady flow conditions can be used in the unsteady pressure and flow analysis. The expression for the glottal discharge coefficient $D_{g}$ that is consistent with the empirical flow resistance in (5) is: 


$$
D_{g} \equiv \frac{\Delta P}{Q_{0}^{2}}=\frac{\rho}{2 C(R e)^{2} A_{g}^{2}}
$$

where $A_{g}=A_{g}(t)$ is the area at the valve (the glottal area), and $Q_{0}$ is the volumetric flow rate.

\subsection{The valve operation}

The glottis is reduced to a punctual dynamic valve which periodically blocks the airway in the pipeline (when vocal folds collide). For the sake of consistency, let us adopt the same area function used to determine the discharge coefficient $D_{g}$ experimentally. The orifice area is approximated in [17] by the equation:

$$
A_{g}(t)= \begin{cases}A_{g \max } \sin (\omega t) & \text { if } \omega t_{0}<\omega t<\omega t_{0}+\pi \\ 0 & \text { if } \omega t_{0}+\pi<\omega t<\omega t_{0}+2 \pi\end{cases}
$$

where $A_{g \max }=15 \mathrm{~mm}^{2}$ and $t_{0}$ is the opening time of the orifice at the beginning of the duty cycle.

This area waveform corresponds to a valve mechanics with one degree of freedom. The area waveform seen by a flow encountering a valve which flaps with a phase lag between the upper and low vocal fold margins as in two-mass vocal fold models $\left(A_{g}^{M 2 M}(t)\right)$ may be adopted in its place, if one desires to represent a more realistic valve mechanics (with two degrees of freedom) [12].

\subsection{The inclusion of feedback}

Vocat tract feedback is the name given to the retroaction of pressure fluctuations in the vocal tract on the flow through the vocal folds. A combination of the law prescribing the dynamics of the valve operation and the valve discharge coefficient suffices to determine the glottal flow which enters the supraglottal duct. This value can be easily updated to include the pressure reverberations occurring in the pipeline, before it is reconsidered in the following step of the time marching numerical algorithm which solves for the flow.

This procedure is standard in vocal fold models which aim at producing realistic flow signals. In order to adapt the water hammer theory to the problem we are discussing, we will make allowances to work with or without feedback. 
Just as in conventional two-mass models, including feedback will introduce qualitative differences in the resulting flow waveforms.

\section{Results}

The results of the basic water hammer analysis applied to the system in figure 1 and under the conditions detailed in the previous section, are presented in figures 2 and 3. Figure 2 shows the intra-tracheal and intra-oral pressure fluctuations during a glottal cycle, which corresponds in this context to a single open-close manœuvre of the vocal valve, associated to the prescription of the orifice area (dashed line).

From the very first cycle, the glottal valve sets up pressure waves that propagate upstream and downstream along the trachea and the vocal tract respectively (rarefaction and compression in the subglottal and supraglottal regions). This pattern is repeated at a frequency of $100 \mathrm{~Hz}$. The pressure drop at the glottal valve is clearly visible. Figure 3 shows the airflow velocity along the pipeline corresponding to the same glottal cycle. The discontinuity in $U(z)$ before the lips corresponds to the narrower $2 \mathrm{~cm}$ section used in the analysis.

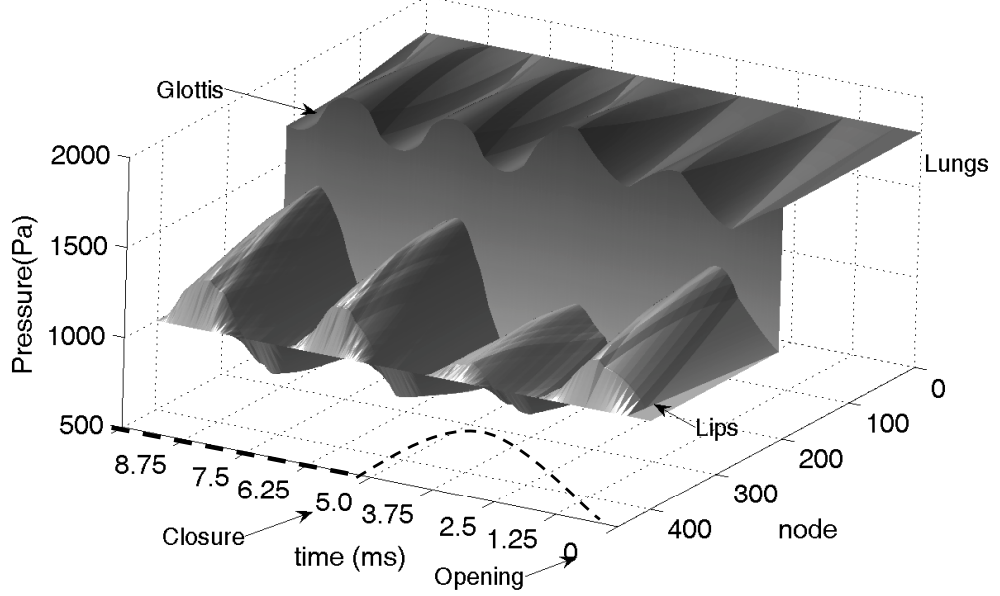

Fig. 2. Basic water hammer prediction of intra-tracheal and intra-oral pressure fluctuations induced by vocal fold motion during a glottal cycle, with a time step of $2.5 \times 10^{-6}$ and 404 nodes covering the $z$ axis. The dashed line is the glottal area function $A_{g}(t)$ in arbitrary units.

In order to compare these results with those obtained with a standard vocal fold model, it is possible to perform the same water hammer analysis with a valve operation law in which the area function corresponds to the minimal section of the glottal channel determined by the two-mass positions calculated by the a two-mass vocal fold model. In general, two-mass models predict 


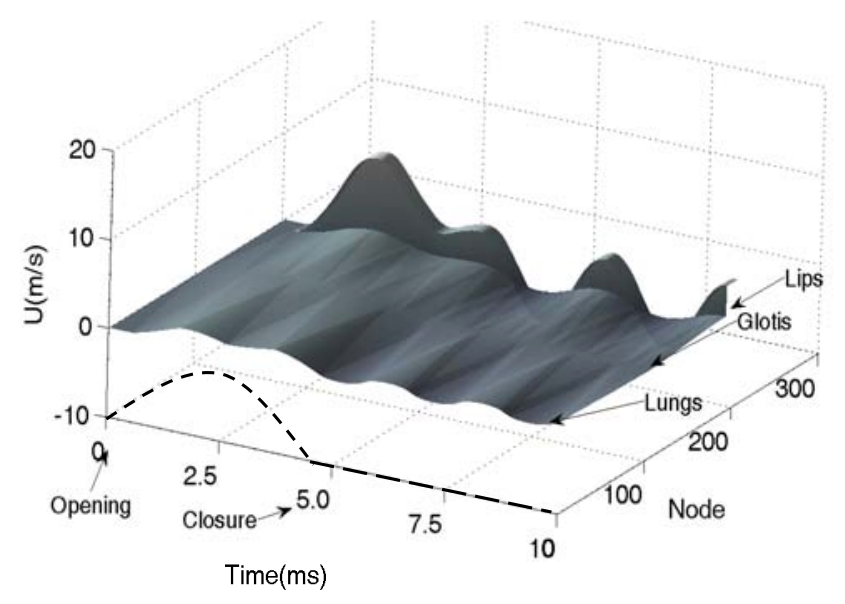

Fig. 3. Basic water hammer prediction of the airflow evolution throughout the vocal system, with a time step of $2.5 \times 10^{-6}$ and 404 nodes covering the $z$ axis. The dashed line is the glottal area function $A_{g}(t)$ in arbitrary units.

a (relatively slow) oscillation onset of the vocal folds, but after this initial transient the mass positions that determine the glottal profile (and hence the area at the glottis) oscillate periodically with a waveform $A_{g}^{M 2 M}(t)$. This area waveform is slightly skewed with respect to the sinusoidal waveform $A_{g}(t)$ of expression (7).

A comparison between the predictions of both models is shown in figure 4 . The two-mass model we are using to calculate the grey functions is the symmetrical version without subglottal loading presented in [16] and analysed in [32]. The parameters controlling the vocal folds in the two-mass model (masses, tensions, rigidity) are set to obtain a signal of $100 \mathrm{~Hz}$ for a subglottal pressure of 800 $P a$, in accordance with the settings adopted for the water hammer flow. The vocal tract configuration in the two-mass model is also chosen to coincide with that of figure 1 .

Notice that the initial transient in the two-mass model is essentially a structural transient, associated to the time needed for the mechanical system to attain self-sustained oscillations: it takes about two cycles for the modelled vocal folds to start oscillating regularly. Instead, the initial transient in the water hammer predictions is a fluid transient, associated to the time needed for the feedback effects from trachea and tract to define a stable glottal flow waveform. This stable regime is attained after one cycle. Beyond this initial transient, both curves (pressure and flow) are in good agreement with the predictions of the vocal fold model. The small differences between the water hammer and the two-mass model flow curves is basically due to the absence of subglottal load in the two-mass model. Note that the glottal flow waveform is slightly more irregular for the water hammer flow, in agreement with this 


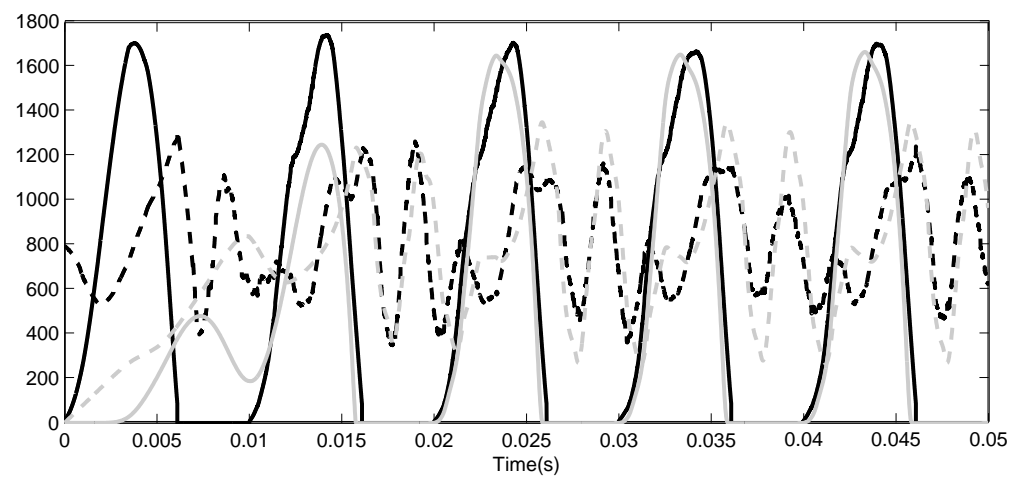

Fig. 4. Pressure (dashed line) and flow (full line) at the glottal exit obtained with a two-mass vocal model (grey) and with a water hammer analysis (black) for a vocal tract configuration corresponding to figure 1. Units are $P a$ for the pressure and dimensionless (Reynolds number) for the flow.

'double feedback' from trachea and vocal tract. Let us also recall that the water hammer flow is calculated with an experimental glottal discharge coefficient, which explains the small departure between both pressure curves. The effect of feedback on the flow signals is often evaluated by visualizing the formant ripples in the glottal flow derivative (see figure 5). These ripples are not smoothed because the effects of friction are neglected in basic water hammer. As expected, they vanish altogether if source-filter separation is enforced, i.e. if the feedback process detailed in section (4.4) is forbidden.

Finally, figure 5 also shows how water hammer flow is sensitive to the glottal valve operating law, and in particular to the symmetry of the area waveform with respect to the opening and closing phases. This confirms that the output in the water hammer case will be strongly dependent on the characterization of the valve. In view of these results, it would also be interesting to prescribe an experimentally determined glottal area waveform, obtained for instance by videostrobolaryngoscopy [33].
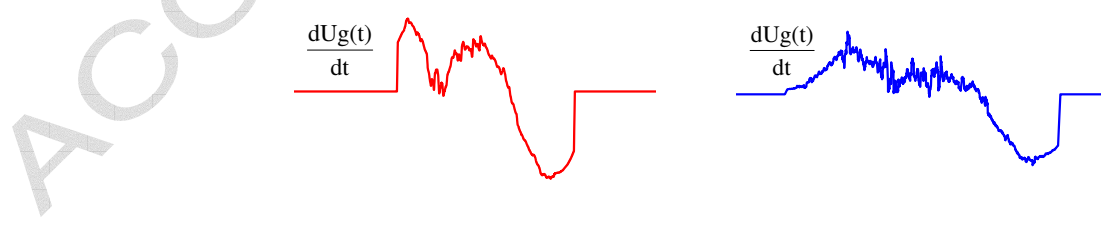

(a)

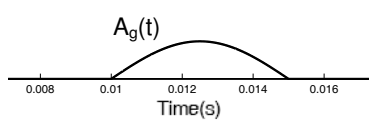

(b)

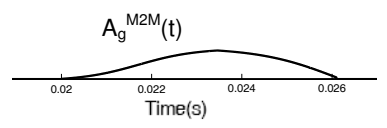

Fig. 5. Glottal flow derivative resulting from a water hammer analysis operated by two different valve laws: the piecewise sinusoidal $A_{g}(t)$ (a) and the skewed area waveform $A_{g}^{M 2 M}(t)$ obtained with a two-mass model. 


\section{Conclusion and perspectives}

In this work, we resume the observation made by Ishizaka in 1976 that water hammer theory can be relevant to describe certain aspects of voice production, and we consider how this analysis of flow transients can be applied to predict simultaneously intra-tracheal and intra-oral pressure and flow within the phonatory system.

We present a brief outline of water hammer theory at different levels of complexity and sketch some guidelines for its applicability to the case of phonation. It is argued that the extended water hammer theory could provide an interesting perspective to analyse voice production in a more realistic basis, including for instance, fluid-structure interaction and articulatory dynamics, while in its simplest version, the water hammer approach is basically equivalent to that of simple physics-based voice production models. This work focuses on the application of the basic water hammer equations to a pipeline system with settings and conditions similar to those adopted in simplified vocal fold models.

The phonatory system is presented as a pipeline fed by an infinite reservoir (the lungs) and intercepted by a valve (the glottis) which opens and closes repeatedly (vocal fold vibration) causing rapid changes in the flow conditions. The analysis reproduces the typical pressure and flow waveforms in the case of a sustained vowel.

From a conceptual point of view, this basic water hammer analysis confirms in a straightforward manner that the main contribution to sound pressure in voice production is the result of the flow perturbations caused by the action of the glottal valve, which explains why the assumption of an acoustic monopolar source used in simplified vocal fold models is a good approximation to the vocal sound source.

The water hammer theory provides an effective tool to predict sound generation, transmission and reflection throughout the phonatory system in a single analysis and with a well established procedure. Since the theory is not restricted to the simple case we have discussed, and is supported by a large body of work on applications involving problems of a degree of complexity which is comparable to that encountered in the speech production system, this work provides the basis for future research in this direction.

\section{References}

[1] R. Skalak, An extension of the theory of waterhammer, Transactions of the ASME 78 (1956) 105-116. 
[2] A. . S. . Tijsseling, Fluid-structure interaciton in liquid-filled pipe systems: A review, Journal of Fluids and Structures 10 (1996) 109146.

[3] D. Chungcharoen, Genesis of Korotkoff sounds, Am. J. Physiol. 207 (1964) 190-194.

[4] J. Allen, T. Gehrke, J. O. . Sullivan, S. T. King, A. Murray, Characterization of the Korotkoff sounds using joint time-frequency analysis, Physiol. Meas. 25 (2004) 107-117.

[5] H. S. Chang, H. Nakagawa, Hypothesis on the pathophysiology of syringomyelia based on simulation of cerebrospinal fluid dynamics, Journal of Neurology Neurosurgery and Psychiatry 74 (2003) 344-347.

[6] N. H. Fletcher, Autonomous vibration of simple pressure-controlled valves in gas flows, J. Acoust. Soc. Am. 93 (4) (1993) 2172-2180.

[7] K. Ishizaka, M. Matsudaira, T. Kaneko, Input acoustic-impedance measurement of the subglottal system, J. Acoust. Soc. Am. 60 (1) (1976) 190-197.

[8] G. C. Burnett, Method and apparatus for voiced speech excitation function determination and non-acoustic assisted feature extraction, United States Patent 20020099541 (2002) A1.

[9] F. Alipour, R. C. Scherer, Flow separation in a computational oscillating vocal fold model, J. Acoust. Soc. Am. 116 (3) (2004) 1710-1719.

[10] J. C. Lucero, A theoretical study of the hysteresis phenomenon at vocal fold oscillation onset-offset, J. Acoust. Soc. Am. 105 (1) (1999) 423-431.

[11] A. Hirschberg, Introduction to aero-acoustics of internal flow, Lecture series von Karman Institute for fluid dynamics ISSN 0377-8312 2 (2001) A1-A133.

[12] K. Ishizaka, J. L. Flanagan, Synthesis of voiced sounds from a two-mass model of the vocal cords, Bell. Syst. Tech. J. 51 (1972) 1233-1268.

[13] X. Pelorson, A. Hirschberg, R. R. van Hassel, A. P. J. Wijnands, Theoretical and experimental study of quasisteady-flow separation within the glottis during phonation. Application to a modified two-mass model, J. Acoust. Soc. Am. 96 (6) (1994) 3416-3431.

[14] D. Sciamarella, P. L. Quéré, Solving for unsteady airflow in a glottal model with immersed moving boundaries, European Journal of Mechanics / B Fluids 27 (1) (2007) 42-53.

[15] I. Titze, Principles of Voice Production, Prentice-Hall, Englewood Cliffs, NJ, 1993.

[16] N. J. C. Lous, G. C. J. Hofmans, R. N. J. Veldhuis, A. Hirschberg, A symmetrical two-mass vocal-fold model coupled to vocal tract and trachea, with application to prosthesis design, Acta Acustica united with Acustica 84 (6) (1998) 11351150 . 
[17] L. Mongeau, N. Franchek, C. H. Coker, R. A. Kubli, Characteristics of a pulsating jet through a small modulated orifice, with application to voice production, J. Acoust. Soc. Am. 102 (1997) 1121-1133.

[18] N. Joukowsky, Uber den hydraulischen stoss in wasserleitungsrohren, Mémoires de l'Académie Impériale des Sciences de St. Pétersbourg Series 8, 9 (1900) 5.

[19] E. J. Blade, W. Lewis, J. H. Goodykoontz, Study of a sinusoidally perturbed flow in a line including a 90 elbow with flexible supports, Washington : National Aeronautics and Space Administration, Technical Note D-1216.

[20] A. F. D'Souza, R. Oldeburger, Dynamic response of fluid lines, ASME Journal of Basic Engineering 86 (1964) 589-598.

[21] D. J. Wood, A study of the response of coupled liquid flow-structural systems subjected to periodic disturbances, ASME Journal of Basic Engineering 90 (1968) 532-540.

[22] E. B. Wylie, V. L. Streeter, Fluid Transients in Systems, Prentice-Hall: Englewood Cliffs, 1993.

[23] M. S. Ghidaoui, M. Zhao, D. A. McInnis, D. H. Axworthy, A review of water hammer theory and practice, Appl. Mech. Rev. 58 (2005) 49-76.

[24] V. L. Streeter, E. B. Wylie, Hydraulic Transients, New York : McGraw-Hill, 1967.

[25] V. L. Streeter, E. B. Wylie, Waterhammer and surge control, Annual Review of Fluid Mechanics 6 (1974) 57-73.

[26] M. Zhao, M. S. Ghidaoui, Efficient quasi-two-dimensional model for water hammer problems, J. Hydr. Engrg. 129 (12) (2003) 1007-1013.

[27] M. Zhao, M. S. Ghidaoui, A. A. Kolyshkin, Perturbation dynamics in unsteady pipe flows, J. Fluid Mech. 570 (2007) 129-154.

[28] T. L. Miller, A. R. Altman, T. Tsuda, T. H. Shaffer, An ultrasound imaging method for in vivo tracheal bulk and youngs moduli of elasticity, Journal of Biomechanics 40 (2007) 16151621.

[29] V. Hermawan, A. M. Al-Jumaily, M. Fatemi, Three non-destructive methods of the modulus of elasticity of the trachea, ASME, International Mechanical Engineering Congress and Exposition, Bioengineering Division IMECE200459091.

[30] K. H. J. Dang, Construction and control of a physiological articulatory model, J. Acoust. Soc. Am. 115 (2) (2004) 853-870.

[31] J. H. Spurk, Fluid Mechanics, Springer-Verlag, 1997, p. 275.

[32] D. Sciamarella, C. d'Alessandro, On the acoustic sensitivity of a symmetrical two-mass model of the vocal folds to the variation of control parameters, Acta Acustica 90 (2004) 746-761. 
[33] P. Woo, Quantification of videostrobolaryngoscopic findings-measurements of the normal glottal cycle, Laryngoscope 106 (3) (1996) 79:1-27. 